\title{
Evaluación y manejo de primera crisis epiléptica
}

Assessment and management of the first epileptic seizure

\author{
Manuel Herrera $^{1}$, Alejandro Escalaya ${ }^{2}$, Ana Suller-Marti ${ }^{1}$, Marco A Vasquez ${ }^{2}$, Juan Toro ${ }^{1}$, Jorge G Burneo ${ }^{1}$

\section{RESUMEN}

Las crisis epilépticas son una causa frecuente de consulta en la emergencia y en la atención ambulatoria. La evaluación de una primera crisis epiléptica reviste gran trascendencia en este contexto, ya que la ocurrencia de ésta no implica necesariamente el diagnóstico de epilepsia (dos o más crisis no provocadas separadas por más de 24 horas; una crisis única con alto riesgo de recurrencia ( $>60 \%)$; o la evidencia de un síndrome epiléptico - definición de la Liga Internacional para la Lucha Contra la Epilepsia, ILAE) y el tratamiento subsecuente. Por otro lado, no todo paciente con primera crisis debe ser dejado en observación sin recibir el manejo apropiado. Esta decisión está en función del riesgo de recurrencia de crisis. Para ello, la Academia Americana de Neurología (AAN de sus siglas en inglés) recomienda la clasificación de la primera crisis epiléptica en cinco grupos y dependiendo del riesgo de recurrencia de crisis establecido para cada uno de ellos, se tendrá una guía para proceder o no con el tratamiento antiepiléptico. Los grupos son: pacientes con crisis epiléptica provocadas; pacientes con crisis sintomáticas agudas; pacientes con crisis sintomática remotas; primera crisis asociada a síndromes epilépticos; primera crisis de causa desconocida. La guía publicada por AAN en el 2015 para el manejo de primera crisis, sugiere que los pacientes con crisis sintomáticas remotas (lesiones cerebrales pasadas no evolutivas), pacientes con anormalidades epileptiformes interictales; pacientes con estudios de imagen anormales (RMN y TC); y pacientes con crisis nocturnas, tienen un riesgo elevado para recurrencia de crisis $(>60 \%)$ por lo que deben ser tratadas. La evidencia disponible a la fecha sugiere también que no hay diferencia significativa en el inicio precoz o diferido del tratamiento antiepiléptico para el control de las crisis a largo plazo.

PALABRAS CLAVE: Convulsiones, epilepsia, recurrencia. (Fuente: DeCS BIREME).

\section{SUMMARY}

Epileptic seizures are a common cause of medical consultation in the emergency room and in outpatient settings. The evaluation of the first epileptic seizure is of upmost importance as not all patients presenting with seizures have epilepsy (two or more unprovoked crises separated more than 24 hours; one single crisis with a high risk of recurrence $(>60 \%)$; or evidence of an epileptic syndrome needing treatment based on the definition by the International League against Epilepsy). On the other hand, not every patient with a first episode should be just observed not offering proper treatment. This decision is based on the risk of recurrence. For that purpose, the American Academy of Neurology (AAN) recommends classifying the first seizures into five groups depending on the risk of recurrence, these groups are: patients with provoked seizures; patients with acute symptomatic seizures; patients with remote symptomatic seizures; first seizure associated with an epileptic syndrome, and first seizure of unknown origin. The AAN guidelines for the management of the first seizure published in 2015 suggests that patients with symptomatic

1 Programa de Epilepsia, Departamento de Ciencias Neurológicas, Schulich School of Medicine and Dentistry, Western University. London, Ontario, Canadá.

2 Departamento de Neurología, Hospital Nacional Cayetano Heredia. Lima, Perú 
remote seizures (non-evolutive and old cerebral lesions), patients with interictal epileptiform abnormalities, patients with abnormal findings on MRI or CT scan, and patients with nocturnal seizures had a high risk for recurrence $(>60 \%)$ and should be treated. Current evidence suggests that there is no difference in early or delay treatment for controlling seizures at the long-term.

KEY WORDS: Seizures, epilepsy, risk factor for seizure recurrence, remote symptomatic seizure, acute symptomatic seizure.

\section{INTRODUCCIÓN}

La epilepsia es un desorden neurológico crónico caracterizado por la recurrencia de crisis epilépticas no provocadas separadas por más de 24 horas, según la Liga Internacional para Lucha Contra la Epilepsia (ILAE por sus siglas en inglés). Aunque la última definición considera también el riesgo alto (mayor al 60\%) de recurrencia de crisis, o la evidencia de un síndrome epiléptico para establecer el diagnóstico después de un primer evento (1).

Un estudio epidemiológico en Latinoamérica, indicó que la prevalencia de epilepsia era similar en los países de la región, de 17,8 por cada 1000 personas (2). Dentro de este estudio se incluyó uno realizado en Perú, donde se incluyeron poblaciones urbanas y pediátricas y se obtuvo una prevalencia de 11,9 por cada 1000 individuos (3). Esta tasa es más alta que en Europa, EEUU y Canadá. Esto quizás se deba a ciertas etiologías que son más prevalentes en el Perú, como la neurocisticercosis y otros procesos infecciosos bacterianos y parasitarios, así como los traumatismos cráneo-encefálicos $(4,5)$.

Si bien se sabe que la epilepsia tiene una alta prevalencia, no se tienen estudios epidemiológicos de primera crisis en el Perú. En países como EEUU, se describe que la incidencia y prevalencia de la primera crisis es mayor que la de epilepsia, entonces uno podría asumir que el mismo escenario se produce en el Perú (6). En el Perú se estima que la epilepsia es el segundo trastorno neurológico más frecuente después de la cefalea, cuya incidencia oscila alrededor de 0,5 a 1,5 por cada 100 habitantes (5). De ello se desprende que la consulta por primera crisis tanto en emergencia y consulta externa representa una casuística importante, no estimada hasta el momento.

Por lo mencionado, resulta relevante el adecuado enfoque y manejo de la primera crisis epiléptica, que estará en función de la correcta identificación del riesgo de recurrencia. Un diagnóstico erróneo de epilepsia no sólo expondría al paciente al estigma que aún existe en nuestra sociedad en contra de los que la padecen, sino también a los posibles efectos adversos a corto y largo plazo de los fármacos antiepilépticos (FAE), además de los costos que implica para el sistema de salud.

A continuación, se presentan ciertas pautas para el manejo del paciente que se presenta a la sala de emergencia o consultorio externo después de una primera crisis epiléptica. En este manuscrito, no se considera el manejo de aquel paciente que se presenta con estatus epiléptico.

\section{1. ¿Crisis o no crisis?}

Esta es la primera pregunta que uno debe formularse y para ello debe tenerse en cuenta el concepto de crisis epiléptica, definida como la aparición transitoria de signos y/o síntomas debido a una actividad neuronal excesiva y/o sincrónica en el cerebro (1).

Enalgunos de los pacientes que sufren de una primera crisis epiléptica, la información semiológica permite un adecuado diagnóstico (6,7). Se debe corroborar el inicio abrupto y duración breve (paroxismal) del episodio, procurar obtener información sobre síntomas motores, sensitivos, sensoriales, cognitivos, el estado de conciencia durante el episodio y síntomas adicionales durante el periodo inmediato posterior al evento. Si hay alta sospecha de crisis epiléptica es fundamental determinar si efectivamente es el primer episodio, o si es que el paciente presentaba previamente episodios de mioclonías, eventos como de "ausencia" o confusión, heridas en la lengua al despertar u otros elementos que el paciente puede no haber identificado o soslayado, y que evidencien crisis pre-existentes y por tanto epilepsia (7).

Las entidades que se confunden con crisis epilépticas se denominan trastornos paroxísticos no epilépticos (TPNE), que se definen como episodios que pueden ser de origen neurológico o no, y que pueden tener una presentación brusca y recurrente. Estas 
pueden manifestarse como movimientos anómalos, alteración del tono corporal, aparentes cambios en la conciencia o alteración de la conducta sin tener una naturaleza epiléptica (8).

Entre los eventos paroxismales de origen no neurológico, el más frecuente es el síncope. $(8,9)$ Existen varios tipos de síncope, de los cuales el más común es el vaso-vagal. El síncope vaso-vagal se puede caracterizar por la presencia de una visión borrosa, visión en túnel, tinnitus, náuseas, palidez y frialdad cutánea. El episodio puede progresar a un trastorno de conciencia y presentar un componente motor tónico-clónico (síncope convulsivo) que representa una respuesta a la deprivación de oxígeno en el cerebro, particularmente el tronco encefálico, sufrida en ese momento. En estos episodios la persona puede inclusive morderse la lengua y relajar los esfínteres. Por lo general, al final del evento la recuperación es relativamente rápida, sin confusión, pero puede haber amnesia de lo ocurrido $(8,9)$.

Otras posibles entidades que deben ser consideradas son la migraña, el ataque isquémico transitorio, los trastornos del movimiento como tics y distonías, y la amnesia global transitoria. Mención especial merecen los trastornos somatomorfos, también denominados trastornos funcionales entre los que destacan las crisis o eventos no epilépticos psicógenos, y las fugas disociativas principalmente. Los cuadros gananciales también deben ser considerados en el diagnóstico diferencial (tabla 1). Si el episodio ocurrió mientras el paciente dormía, entonces es importante considerar los trastornos del sueño o parasomnias $(8,9,10)$.

$\mathrm{Si}$ el episodio evaluado se considera crisis epiléptica entonces se debe continuar con la siguiente pregunta.

\section{2. ¿Qué tipo de crisis epiléptica?}

Una vez que se determine que el evento fue una crisis epiléptica, es importante tipificarla. Para esto uno debe remitirse a la clasificación de crisis del 2017 propuesta por la Liga Internacional en contra de la epilepsia (ILAE, por sus siglas en inglés) en la que se establecen tres categorías: crisis de inicio focal, de inicio generalizado y de inicio desconocido (tabla 2)

Tabla 1. Diagnóstico diferencial de crisis epilépticas.

\begin{tabular}{|c|c|c|c|c|}
\hline Tipo de evento & $\begin{array}{l}\text { Síntomas } \\
\text { premonitorios }\end{array}$ & $\begin{array}{l}\text { Características del } \\
\text { episodio }\end{array}$ & Duración promedio & $\begin{array}{l}\text { Síntomas post- } \\
\text { episodio }\end{array}$ \\
\hline $\begin{array}{l}\text { Crisis no epiléptica } \\
\text { psicógena }\end{array}$ & Variable & $\begin{array}{l}\text { Capacidad de } \\
\text { respuesta variable, } \\
\text { no estereotipada, } \\
\text { movimientos inusuales, } \\
\text { labilidad emocional }\end{array}$ & $\begin{array}{l}\text { Muy variable. Suele } \\
\text { ser prolongada }(>5 \\
\text { minutos) }\end{array}$ & $\begin{array}{l}\text { Variable, con } \\
\text { frecuencia ninguno }\end{array}$ \\
\hline Síncope & $\begin{array}{l}\text { Frecuentes: mareos, } \\
\text { sensación de } \\
\text { desvanecimiento }\end{array}$ & $\begin{array}{l}\text { Caída, frecuente cierre } \\
\text { ocular, movimientos } \\
\text { variables o tónico- } \\
\text { clónicos (síncope } \\
\text { epiléptico) }\end{array}$ & $\begin{array}{l}\text { Por lo general } \\
\text { menos de } 1 \text { minuto }\end{array}$ & $\begin{array}{l}\text { Variables, con } \\
\text { frecuencia ninguno }\end{array}$ \\
\hline Migraña & $\begin{array}{l}\text { Aura con alteración } \\
\text { sensorial prolongada } \\
\text { (20-60minutos) }\end{array}$ & $\begin{array}{l}\text { Cefalea hemicránea, } \\
\text { pulsátil u opresiva de } \\
\text { intensidad progresiva, } \\
\text { asociada a nauseas, } \\
\text { fotofobia, etc. }\end{array}$ & Horas o días & $\begin{array}{l}\text { Cansancio, } \\
\text { somnolencia, } \\
\text { desconcentración }\end{array}$ \\
\hline $\begin{array}{l}\text { Ataque isquémico } \\
\text { transitorio }\end{array}$ & No específicos & $\begin{array}{l}\text { Déficit focal agudo y } \\
\text { transitorio }\end{array}$ & $\begin{array}{l}\text { Menos de } 24 \text { horas, } \\
\text { usualmente }<60 \\
\text { minutos }\end{array}$ & Ninguno \\
\hline Parasomnia & Ninguno & $\begin{array}{l}\text { Vocalización, confusión, } \\
\text { deambulación }\end{array}$ & Minutos & Amnesia, confusión \\
\hline Cataplexia & $\begin{array}{l}\text { Emociones intensas } \\
\text { principalmente } \\
\text { en contexto de } \\
\text { narcolepsia }\end{array}$ & $\begin{array}{l}\text { Atonía muscular, } \\
\text { conciencia preservada o } \\
\text { ataque de sueño }\end{array}$ & Segundos a minutos & Ninguno \\
\hline
\end{tabular}

Modificada de: St. Louis EK, Cascino GD. Diagnosis of Epilepsy and Related Episodic Disorders. Continuum. 2016; 22(1): 15-37 
Tabla 2. Clasificación de tipos de crisis epilépticas de la Liga Internacional en Contra de la Epilepsia.

\begin{tabular}{|c|c|c|}
\hline Inicio Focal & $\begin{array}{l}\text { - Sin alteración de la } \\
\text { conciencia }\end{array}$ & Início motor \\
\hline \multirow{9}{*}{ Inicio generalizado } & - Con alteración de la conciencia & $\begin{array}{l}\text { Automatismos, atónico, clónico, espasmos } \\
\text { epilépticos, hipercinéticos, mioclónicos, tónicos }\end{array}$ \\
\hline & $\begin{array}{l}\text { - Focal que evoluciona a } \\
\text { bilateral tónico-clónica }\end{array}$ & - Inicio no motor \\
\hline & & $\begin{array}{l}\text { - Autonómico, alteración del comportamiento, } \\
\text { cognitivo, emocional, sensitivo }\end{array}$ \\
\hline & & - Tónico-clónico \\
\hline & & - Clónico \\
\hline & - Motor & - Tónico \\
\hline & & - Mioclónico \\
\hline & & - Atónico \\
\hline & - No motor & - Ausencia típica, atípica \\
\hline \multirow[t]{2}{*}{ Inicio desconocido } & - Motor & $\begin{array}{l}\text { - Tónico clónico } \\
\text { - Espasmos epilépticos }\end{array}$ \\
\hline & - No motor & -Alteración del comportamiento \\
\hline No clasificada & & \\
\hline
\end{tabular}

(11). En esta clasificación hay un apartado denominado "no clasificables" en el cual se agrupan los casos en los que se obtuvo la mayor información posible sobre el inicio del evento, pero es insuficiente para asignarlo a una de las tres categorías (11).

En esta última clasificación, algunos términos tienen una equivalencia con algunas denominaciones clásicas de la clasificación de 1981. Así, una crisis de inicio focal sin alteración de conciencia es equivalente al término clásico "crisis parcial simple", y cuando tiene alteración de conciencia es equivalente a la antigua "crisis parcial compleja". Igualmente, la crisis focal que evoluciona a bilateral tónicoclónica previamente se denominaba "crisis parcial secundariamente generalizada" (12).

Esta clasificación se considera columnar, pero no jerárquica, por lo que se puede saltar niveles entre la descripción del compromiso motor (presente o ausente) y la descripción del estado de conciencia (alterada o preservada). Si no se conociese si una crisis focal tuvo o no alteración de conciencia, se puede omitir dicho detalle al clasificarla $(11,12)$.

La mayoría de los pacientes que asisten a la emergencia o al consultorio externo, lo hacen tras tener una crisis tónico-clónica generalizada (13). Pero es importante determinar si la crisis fue focal con posterior evolución a una crisis tónico-clónica bilateral o si se trata de una crisis tónico-clónica generalizada desde su inicio. Las siguientes consideraciones ayudarían en la clasificación:

a. La edad del paciente provee cierta orientación, ya que es más probable que las crisis focales que progresen a crisis tónico-clónica bilateral sucedan en pacientes adultos y ancianos que en adolescentes, niños, infantes y neonatos (14). Aunque no haya una descripción de aura, es aún importante considerar el inicio focal ya que la propagación eléctrica de una crisis puede ser muy rápida y no estar acompañada de claras manifestaciones clínicas en su origen. Un típico ejemplo de propagación rápida son las crisis de origen occipital, en las cuales las descargas epilépticas se propagan con mucha frecuencia y mucha rapidez a la región temporal o a la frontal, esta última con gran tendencia a la progresión tónico-clónica.

b. La presencia de un aura sugiere una crisis de inicio focal. En algunos casos el aura no es descrita espontáneamente por el paciente o hay amnesia del inicio del evento (15).

c. La presencia de eventos previos que pueden corresponder a crisis epilépticas, los cuales en algunos casos no fueron necesariamente similares al episodio que llevó al paciente a la consulta. Ejemplo de ellas son las mioclonías del despertar. Estas pueden ocurrir de manera espontánea sin que sean seguidas de crisis tónico-clónica generalizadas. 
Sin embargo, en pacientes con epilepsia generalizada, las mioclonías pueden ocurrir en salvas previo a una crisis tónico-clónica generalizada. Por otro lado, episodios de déjà-vu o jamais- $v u$, alucinaciones auditivas, gustatorias, $\mathrm{u}$ olfatorias que ocurren de manera estereotipada y sin estar asociadas a la afectación de la de conciencia, darían más apoyo a la posibilidad de que lo que ocurrió no fue un evento aislado, sino que el paciente sufrió una crisis focal que luego progreso a una crisis tónico-clónica bilateral (7).

d. El hallazgo de un déficit neurológico focal puede indicar una potencial lesión estructural epileptogénica que nos conmina a re-interrogar al paciente en busca de signos, tal vez sutiles, que sugieran un inicio ictal compatible con el foco detectado en el examen (1).

\section{3. ¿Crisis provocada o no?}

Antes de considerar este análisis es conveniente exponer la clasificación de la primera crisis en cinco grupos que permitirá tener un mejor análisis del riesgo de recurrencia de crisis $\mathrm{y}$, por tanto, la conducta terapéutica a seguir (7).
- Crisis epiléptica provocada

- Crisis sintomática aguda

- Crisis sintomática remota

- Crisis epiléptica asociada a síndromes epilépticos

- Crisis sin causa identificada

Se define como crisis epiléptica provocada a aquella debida a una causa claramente identificable como medicación, abuso de sustancias, cuadros metabólicos agudos, intoxicaciones, etc. (crisis febriles no serán consideradas en el presente artículo a pesar de estar incluidas en esta categoría) (7). Algunos autores consideran en este grupo a las lesiones cerebrales agudas; sin embargo, en la presente revisión seguiremos las recomendaciones de la AAN y las consideraremos en un grupo distinto denominado el de las crisis sintomáticas agudas (16), las cuales son secundarias a procesos agudos intracraneales como infartos cerebrales, hemorragias intracerebrales, traumatismos encéfalo craneanos severos, infecciones del Sistema Nerviosos Central (encefalitis, meningitis, etc.). Se decidió considerar la independencia de este último grupo debido a la forma en que producen la lesión cerebral y los cambios permanentes que dejan (zonas de gliosis y encefalomalacia) (16).

Tabla 3. Causas frecuentes de crisis epiléptica provocada y sintomática aguda.

\section{CRISIS EPILÉPTICAS PROVOCADAS, causas}

Alteraciones metabólicas

- Hipoglicemia

- Hiperglicemia no cetósica

- Hiponatremia

- Hipernatremia

- Uremia por insuficiencia renal aguda o crónica

- Insuficiencia hepática aguda o crónica

- Hipocalcemia

- Porfiria

- Hipomagnesemia

- Hipofosfatemia

Ciertos fármacos y drogas

Abstención de fármacos y toxinas con propiedades depresoras del sistema nervioso central (benzodiazepinas, alcohol)

Encefalopatía anóxica

\section{CRISIS SINTOMÁTICA AGUDA, causas}

Traumatismo encéfalo-craneano (primeras dos semanas)

Infecciones agudas del sistema nervioso central

- Meningoencefalitis víricas, bacterianas y parasitarias

- Meningitis bacterianas

- Procesos infecciosos localizados (absceso, empiema)

Encefalitis límbicas paraneoplásicas (fase aguda)

Encefalitis autoinmunes (fase aguda) 
El grupo con crisis sintomáticas remotas está caracterizado por pacientes que presentan lesiones cerebrales pre-existentes (no evolutivas) de larga data y que sufren súbitamente sin ningún desencadenante aparente (no provocadas) una primera crisis epiléptica $(17,18)$.

Las crisis asociadas a síndromes epilépticos se dan en un contexto en el que las investigaciones subsecuentes establecen la pertenencia a ciertos síndromes electro-clínicos que permiten diagnosticar al paciente con epilepsia tras la primera crisis (7).

Dado que el diagnóstico de epilepsia y, por ende, la administración de tratamiento con FAE, incluye dos o más crisis epilépticas no provocadas o el riesgo elevado $(>60 \%)$ de tener una segunda crisis después de haber desarrollado una primera (1), es de suma importancia conocer el riesgo de recurrencia por cada grupo de pacientes que presenta una primera crisis (16-18). Diversos estudios en las últimas décadas han estimado que el $10 \%$ de la población general tendrá una crisis epiléptica no provocada en algún momento de su vida (15). De éstas, menos de la mitad desarrollarán un segundo evento, alrededor de un 33\% aproximadamente (19).

Se estima $28 \%$ de riesgo de recurrencia en pacientes que han tenido crisis provocadas (20) (tabla 3). Por lo general estas crisis no requieren de FAE a mediano o largo plazo. Sin embargo, dependiendo del contexto clínico se puede administrar medicación antiepiléptica como parte del manejo sintomático durante la fase aguda, el cual no será necesario una vez que el problema de fondo haya sido corregido (ejemplo, corrección gradual de sodio en casos de hiponatremia sintomática) $(15,20)$.

Para los pacientes con crisis sintomática aguda, que como se mencionó anteriormente, están en relación con lesiones cerebrales agudas, diversos estudios muestran un riesgo de recurrencia (a cinco años) similar para una segunda crisis en función de tres tipos de etiologías principalmente: $33 \%$ para pacientes con infarto cerebral agudo; $13,4 \%$ para aquellos con injuria cerebral traumática; y $16,6 \%$ para aquellos con infecciones del sistema nervioso central (16).

Existen estudios comparativos entre pacientes con primera crisis sintomática aguda versus aquellos con primera crisis sintomática remota, en donde las etiologías analizadas para este segundo grupo fueron también insultos cerebrales debido a infarto cerebral, lesión cerebral traumática e infección del $\mathrm{SNC}$, encontrándose riesgos para recurrencia de crisis de $71,5 \%, 46,6 \%$ y $63,5 \%$, respectivamente (16-18). Además, se analizó el riesgo de mortalidad en los primeros 30 días después de la primera crisis en ambos grupos y encontraron un riesgo incrementado de 8.9 veces para aquellos con crisis sintomática aguda. En conclusión, se demostró que el riesgo de recurrencia de crisis es alto, y que, por lo tanto, se requiere medicación antiepiléptica para aquellos pacientes que presentaron crisis sintomáticas remotas (16-18).

\section{4. ¿Tratar o no tratar?}

Hay que recordar que el objetivo del tratamiento antiepiléptico es tener un control adecuado o ausencia sostenida de crisis, definida por un periodo libre de crisis de un año o la ausencia de éstas por un periodo que sea tres veces más largo al lapso más prolongado entre una crisis y otra que tuvo el paciente (21). Sin embargo, estas consideraciones son importantes para el manejo a largo plazo del paciente ya diagnosticado con epilepsia.

Retomando el tema que nos avoca, el manejo de la primera crisis es importante considerar si el inicio del tratamiento precoz o el diferido tienen una repercusión negativa o positiva en la respuesta al tratamiento a largo plazo.

Asistiendo a esta interrogante, la AAN publicó en el 2015 la Guía para el Manejo de Primera Crisis no Provocada basada en una extensa revisión de la evidencia disponible. En esa revisión se describió que pacientes con una lesión o insulto cerebral previo (sintomáticos remotos), aquellos con anormalidades epileptiformes en el electroencefalograma (EEG), anormalidades significativas en los estudios de imágenes, y aquellos con crisis nocturnas, tienen un alto riesgo de recurrencia $(>60 \%)$ y por tanto deben ser tratados $(18,22,23)$.

La AAN en su guía del 2015, establece también el tratamiento antiepiléptico apropiado y precoz, reduce el riesgo de tener una segunda crisis en un $35 \%$ en los siguientes 2 años; aunque si se retrasa el inicio del tratamiento hasta después de una segunda crisis epiléptica, no hay diferencia significativa en la posibilidad de remisión a largo plazo. $(22,23)$.

La profilaxis en una primera crisis epiléptica no tiene utilidad excepto en el caso de un traumatismo craneoencefálico grave en el periodo post-traumático 
temprano (7). Por lo tanto, la decisión siempre va a ser "tratar o no tratar" a los pacientes que han tenido una única crisis epiléptica. Esta decisión se basará principalmente en el riesgo de tener una segunda crisis epiléptica (18).

Se debe informar a los pacientes que todos los fármacos antiepilépticos tienen efectos adversos (con un riesgo que va del 7 al 31\%). Se les debe mencionar los específicos del fármaco que se les está prescribiendo, siendo la mayoría, leves y reversibles con la retirada de la medicación $(22,23)$.

\section{Cuando el cuadro no es claro}

Cuando no se tiene la certeza de que el episodio paroxístico que llevó al paciente a emergencia fue una crisis epiléptica, aún después de la obtención de exámenes auxiliares como las neuroimágenes y el EEG, se debe hacer un seguimiento del paciente. Hay que recordar que el EEG puede ser normal en pacientes con epilepsia $(11,12)$.

La utilización de técnicas como la deprivación de sueño antes de realizar un EEG aumentan las posibilidades de poder registrar alguna actividad interictal o incluso una crisis durante la prueba (15).

El monitoreo con video-EEG es una alternativa sumamente importante, pero reservada para pacientes con recurrencia de episodios de difícil caracterización y que generen duda diagnóstica (15).

\section{Consideraciones tras el diagnóstico de una primera crisis epiléptica}

Del total de pacientes que son evaluados por una primera crisis, una cantidad importante serán diagnosticados de manera inmediata o al corto plazo de Epilepsia y a los restantes se les explicará el riesgo bajo pero existente de desarrollar una segunda crisis en los siguientes años (19). Se estima que el $30 \%$ de pacientes con epilepsia sufrirán ansiedad, principalmente aquellos con mal control de crisis (24). La depresión y ansiedad interictal constituyen el principal impacto en la calidad de vida del paciente con epilepsia, incluso más que la frecuencia de crisis (25).

Una consideración sumamente importante son las restricciones que podrían tener estos pacientes tras el diagnóstico como la conducción de vehículos, especialmente si dicha actividad está directamente relacionada con su actividad laboral; el trabajar en las alturas (albañil, electricista, etc.); el nadar sin supervisión o bañarse en tinas, etc. Diversas actividades y situaciones que ameritan una adecuada consejería que debe ser provista por el personal médico que, aunque parezcan simples, pueden ser de vital importancia, como las recomendaciones de no asegurar la puerta del baño cuando se use, tener supervisión permanente si se va a entrar a una piscina, evitar el mar, en la medida de los posible coordinar elementos de seguridad ocupacional o informar a la autoridad correspondiente. Particular importancia reviste las licencias de conducir vehículos que en nuestro país no está regulada de manera estricta.

\section{CONCLUSIONES}

Las crisis epilépticas son motivo frecuente de consulta en urgencias y consulta externa. Es importante la realización de un enfoque preciso en donde es preponderante la determinación del riesgo de recurrencia de crisis. Para ello se debe de obtener la máxima información posible del paciente al igual de las personas que presenciaron el evento, tanto antes, durante como después del evento, acto que debe ser acompañado un examen neurológico completo. Con dicha información se debe decidir si el evento fue una crisis epiléptica o no y definir si efectivamente es un primer evento o no.

Exámenes auxiliares como un registro de EEG y pruebas de imagen que nos ayuden a precisar el diagnóstico -previamente establecido con la información clínica, en grado posible o probable-; pueden aportar información para valorar el riesgo de recurrencia (actividad epileptiforme interictal o imágenes anormales en la resonancia magnética nuclear o tomografía computarizada). Con esta información se determinará si el paciente amerita tratamiento sintomático antiepiléptico a corto plazo mientras se resuelve la causa subyacente (en crisis provocadas) o si el paciente presenta alto riesgo de recurrencia y requerirá medicación a largo plazo. Se elegirá el fármaco más apropiado dependiendo del perfil del paciente y exponiendo los posibles efectos adversos del mismo.

\section{Declaración de financiamiento y de conflictos de intereses:}

El Dr. Jorge Burneo es portador del Jack Cowin Chair in Epilepsy Research de la Western University. También recibe apoyo financiero para el fellowship 
de epilepsia que dirige, de las compañías Eisai y Sunovion Canadá. Además, recibe apoyo financiero para investigación de Western University, Lawson Research Institute (Canada), EpLink (Programa de investigación de Epilepsia del Ontario Brain Institute), y del fondo de apoyo Rick Berg.

Los otros autores no tienen conflictos de interés o alguna declaración de financiamiento.

\section{Correspondencia:}

Dr. Jorge G Burneo

Epilepsy program,

Department of Clinical Neurological Sciences

Schulich School of Medicine, Western University

University Hospital - LHSC, 339 Windermere Rd, B10-120

London, ON, N6A5A5, Canada.

Correo electrónico: Jburneo2@uwo.ca

Teléfono: 15196633464 /Fax: +15196633498

\section{REFERENCIAS BIBLIOGRÁFICAS}

1. Fisher RS, Acevedo C, Arzimanoglou A, Bogacz A, Cross H, Elger CE, et al. Definición clínica práctica de la epilepsia. Epilepsia. 2014; 55(4):475-482.

2. Burneo JG, Tellez-Zentero J, Wiebe S. Understanding the burden of epilepsy in Latin America: A systematic review of its prevalence and incidence. Epilepsy Res. 2005; 66:63-74.

3. Reyes JC, Ignacio ME. Prevalence of epilepsy in childhood (Lima y Callao 1992). Rev Med Inst Peru Segur Soc. 1994; 3: 21-25.

4. Placencia M. Incidencia, prevalencia y magnitud global de las epilepsias en América Latina y el Caribe. En: Campos MG, Kanner AM. Epilepsias Diagnóstico y Tratamiento. Santiago, Chile: Mediterráneo Ltda; 2004.p. 49-68.

5. Ministerio de Salud. RM N ${ }^{\circ} 692-2006 / M I N S A$. Guía de Práctica Clínica de Epilepsia. Lima: Ministerio de Salud; 2015.

6. Gavvala JR, Schuele SU. New-onset seizure in adults and adolescents a review. JAMA. 2016;316(24):26572668.

7. Bergey G. Management of a first seizure. Continuum (Minneap Minn) 2016; 22(1):38-50.

8. Martínez J, Rodríguez X. Diagnóstico diferencial de las crisis epilépticas en la edad pediátrica y adulta. En: Sánchez JC, Ramos J. La epilepsia en situaciones de urgencia. Madrid: Editorial Medica Panamericana; 2017. p. 17-28.

9. David P. Trastornos paroxísticos no epiléptico. Rev Chil Epilepsia. 2006; 7(1):48-54.

10. Pérez J. Trastornos paroxísticos no epilépticos. BSCP Can Ped. 2003; 27(1):25-36.
11. Scheffer IE, Berkovic S, Capovilla G, Connolly MB, French J, Guilhoto L. ILAE classification of the epilepsies: Position paper of the ILAE Comission for Classification and Terminology. Epilepsia. 2017; 58(4):512-521.

12. Fisher RS, Cross JH, French JA, et al. Operational classification of seizure types by the International League Against Epilepsy: Position Paper of the ILAE Commission for Classification and Terminology. Epilepsia. 2017; 58(4):522-530.

13. McKee PJ, Wilson EA, Dawson JA, Larkin JG, Brodie M. Managing seizures in the casualty department. BMJ. 1990; 300(6730):978-9.

14. Faught E, Richman J, Martin R, et al. Incidence and prevalence of epilepsy among older U.S. Medicare beneficiaries. Neurology. 2012; 78(7):448453.

15. St. Louis EK, Cascino GD. Diagnosis of Epilepsy and Related Episodic Disorders. Continuum. 2016; 22(1):15-37.

16. Hesdorffer DC, Benn EK, Cascino GD, Hauser WA. Is a first acute symptomatic seizure epilepsy? mortality and risk for recurrent seizure. Epilepsia. 2009; 50(5):1102Y1108.

17. Krumholz A, Wiebe S, Gronseth G, et al. Practice parameter: evaluating an apparent unprovoked first seizure in adults (an evidence-based review): report of the Quality Standards Subcommittee of the American Academy of Neurology and the American Epilepsy Society. Neurology. 2007; 69(21):19962007.

18. Krumholz A, Wiebe S, Gronseth GS, et al. Evidencebased guideline: management of an unprovoked first seizure in adults: report of the Guideline Development Subcommittee of the American Academy of Neurology and the American Epilepsy Society. Neurology. 2015; 84(16):1705-1713.

19. Hauser WA, Rich SS, Lee JR, et al. Risk of recurrent seizures after two unprovoked seizures. $\mathrm{N}$ Engl $\mathrm{J}$ Med. 1998; 338(7):429-434.

20. Kho LK, Lawn ND, Dunne JW, Linto J. First seizure presentation: do multiple seizures within 24 hours predict recurrence? Neurology. 2006; 67(6):10471049.

21. López F, Rodríguez X, Gil-Nagel A, et al. Epilepsia resistente a fármacos. Concepto y alternativas terapéuticas. Neurología. 2015; 30(7):439-446.

22. Marson A, Jacoby A, Johnson A, et al. Immediate versus deferred antiepileptic drug treatment for early epilepsy and single seizures: a randomised controlled trial. Lancet. 2005; 365(9476):2007-2013.

23. Kim LG, Johnson TL, Marson AG, Chadwick DW; MRC MESS Study group. Prediction of risk of seizure recurrence after a single seizure and early epilepsy: further results from the MESS trial. Lancet Neurol. 2006; 5(4):317-322. 
24. O’Donoghue MF, Goodridge DM, Redhead K, Sander JW, Duncan JS. Assessing the psychosocial consequences of epilepsy: a community-based study. British Journal of General Practice. 1999; 49(440):211-4.

25. Johnson EK, Jones JE, Seidenberg M, Hermann BP. The relative impact of anxiety, depression, and clinical seizure features on health-related quality of life in epilepsy. Epilepsia. 2004; 45(5):544-50.

26. Sánchez JC, Sladogna C. Conceptos Generales. En: Sánchez JC, Ramos J. La Epilepsia en situaciones de urgencia. Madrid: Editorial Medica Panamericana; 2017. p. 1-16.
27. National Institute for Health and Care Excellence. Epilepsies: diagnosis and management: NICE guideline. Washington DC: National Institute for Health and Care Excellence; 2012.

28. Urrestarazu E, Murie M, Viteri C. Manejo de la primera crisis epiléptica y del status en urgencias. An Sist Sanit Navar. 2008; 31 (Supl 1):61-73.

29. Burneo J, Kuzniecky R. Neuroimágenes estructurales y funcionales. En: Campos MG, Kanner AM. Epilepsias Diagnóstico y Tratamiento. Santiago, Chile: Mediterráneo Ltda; 2004.

Recibido: 02/04/2020

Aceptado: 03/08/2020 